\title{
Complex Projection of Quasianti-Hermitian Quaternionic Hamiltonian Dynamics ${ }^{\star}$
}

\author{
Giuseppe SCOLARICI
}

Dipartimento di Fisica dell'Università del Salento, and INFN, Sezione di Lecce, I-73100 Lecce, Italy

E-mail: scolarici@le.infn.it

Received July 05, 2007, in final form September 03, 2007; Published online September 08, 2007

Original article is available at http://www.emis.de/journals/SIGMA/2007/088/

\begin{abstract}
We characterize the subclass of quasianti-Hermitian quaternionic Hamiltonian dynamics such that their complex projections are one-parameter semigroup dynamics in the space of complex quasi-Hermitian density matrices. As an example, the complex projection of a spin- $\frac{1}{2}$ system in a constant quasianti-Hermitian quaternionic potential is considered.
\end{abstract}

Key words: pseudo-Hermitian Hamiltonians; quaternions

2000 Mathematics Subject Classification: 81P68; 15A33

\section{Introduction}

Many years ago, it was shown, by using some lattice theoretic arguments, that it is possible to consider the set of states of a quantum system as a vector space over the real, complex or quaternionic fields [1]. However, the research on quaternionic quantum mechanics (QQM) began much later [2] and pursued up to now. A systematic study of QQM is given in [3]. In this context, some recent studies have been developed along two seemingly uncorrelated lines.

On one hand, after a seminal idea by Kossakowski [4], the complex projection of dynamics generated by (time independent) quaternionic anti-Hermitian Hamiltonians was studied by showing that they represent one-parameter semigroup dynamics in the space of complex (Hermitian) density matrices $[5,6,7,8]$. This peculiarity can be useful, for instance, in the case of two qubits compound system, the complex projection of quaternionic unitary dynamics between pure states permits description of interesting phenomena as decoherence and optimal entanglement generation [8].

On the other hand, motivated by the recent studies on non-Hermitian Hamiltonians [9], pseudoanti-Hermitian quaternionic Hamiltonians were introduced in order to generalize standard anti-Hermitian Hamiltonians in quaternionic Hilbert space [10]. The dynamics generated by such (time independent) Hamiltonians preserves an alternative (in general) indefinite inner product in the Hilbert space. In this context, it was proven that if (and only if) the pseudoantiHermitian quaternionic Hamiltonians belong to the subclass of quasianti-Hermitian ones, then, an alternative positive definite quaternionic inner product preserved by the corresponding dynamics can be introduced [11].

Moreover, it was shown that the complex quasi-Hermitian systems can be described as open quantum systems. Indeed, a master equation of Lindblad-Kossakowski type can be derived for such systems, obtaining one-parameter semigroup dynamics in the space of complex quasiHermitian density matrices [12].

${ }^{\star}$ This paper is a contribution to the Proceedings of the 3-rd Microconference "Analytic and Algebraic Methods III". The full collection is available at http://www.emis.de/journals/SIGMA/Prague2007.html 
Motivated by these three apparently separated topics, we intend to study, in this paper, the complex projection of quasianti-Hermitian quaternionic Hamiltonian dynamics. We will show that if (and only if) the Hamiltonian is $\eta$-quasianti-Hermitian with respect a (Hermitian positive definite) complex $\eta$, then the corresponding complex projection dynamics generates oneparameter semigroup dynamics in the space of complex quasi-Hermitian density matrices. This result, allows us to produce systematically, via the complex projection operation, one-parameter semigroup dynamics in the space of complex quasi-Hermitian density matrices. Moreover, we will obtain the complex projection of quaternionic anti-Hermitian Hamiltonian dynamics as a very particular case of this more general setting.

The plan of the paper is the following: In Section 2 we will introduce the density matrix formalism for quaternionic spaces and discuss the complex projection of anti-Hermitian quaternionic Hamiltonian dynamics. In Section 3 the notion of quaternionic pseudo-Hermitian density matrix is introduced and a corresponding Liouville-von Neumann type equation is derived. Moreover, the complex projection of quasianti-Hermitian quaternionic dynamics is considered in Section 4, where the subclass of quasianti-Hermitian quaternionic Hamiltonian dynamics such that their complex projections are one-parameter semigroup dynamics in the space of complex quasi-Hermitian density matrices is characterized. In order to illustrate these results, the com-

plex projection of a spin- $\frac{1}{2}$ system in a constant quasianti-Hermitian quaternionic potential is studied in the last section.

\section{Complex projection of anti-Hermitian quaternionic Hamiltonian dynamics}

We will discuss, in this section, some results about the complex projection of anti-Hermitian quaternionic Hamiltonian dynamics.

A (real) quaternion is usually expressed as

$$
q=q_{0}+q_{1} i+q_{2} j+q_{3} k
$$

where $q_{l} \in \mathbb{R}(l=0,1,2,3), i^{2}=j^{2}=k^{2}=-1, i j=-j i=k$.

The quaternion skew-field $\mathbb{Q}$ is an algebra of rank 4 over $\mathbb{R}$, non commutative and endowed with an involutive anti-automorphism (conjugation) such that

$$
q \rightarrow \bar{q}=q_{0}-q_{1} i-q_{2} j-q_{3} k
$$

The density matrix $\rho_{\psi}$ associated with a pure state $|\psi\rangle$ belonging to a quaternionic $n$-dimensional right Hilbert space $\mathbb{Q}^{n}$ is defined by [3]

$$
\rho_{\psi}=|\psi\rangle\langle\psi|
$$

and is the same for all normalized ray representatives. By definition, density matrices $\rho_{\psi}$ associated with pure states, are represented by rank one, positive definite quaternionic Hermitian operators on $\mathbb{Q}^{n}$ with unit trace. In analogy with standard quantum mechanics $(\mathrm{CQM})$, quaternionic mixed states are described by positive quaternionic Hermitian operators (density matrices) $\rho$ on $\mathbb{Q}^{n}$ with unit trace and rank greater than one.

The expectation value of a quaternionic Hermitian operator $O$ on a state $|\psi\rangle$ can be expressed in terms of $\rho_{\psi}$ as [3]

$$
\langle O\rangle_{\psi}=\langle\psi|O| \psi\rangle=\operatorname{Re} \operatorname{Tr}(O|\psi\rangle\langle\psi|)=\operatorname{Re} \operatorname{Tr}\left(O \rho_{\psi}\right)
$$


Expanding $O=O_{\alpha}+j O_{\beta}$ and $\rho=\rho_{\alpha}+j \rho_{\beta}$ in terms of complex matrices $O_{\alpha}, O_{\beta}, \rho_{\alpha}$ and $\rho_{\beta}$, it follows that the expectation value $\langle O\rangle_{\psi}$ may depend on $O_{\beta}$ or $\rho_{\beta}$ only if both $O_{\beta}$ and $\rho_{\beta}$ are different from zero. Indeed,

$$
\langle O\rangle=\operatorname{Re} \operatorname{Tr}(O \rho)=\operatorname{Re} \operatorname{Tr}\left(O_{\alpha} \rho_{\alpha}-O_{\beta}^{*} \rho_{\beta}\right),
$$

where $*$ denotes complex conjugation.

Thus, the expectation value of an Hermitian operator $O$ on the state $\rho$ depends on the quaternionic parts of $O$ and $\rho$, only if both the observable and the state are represented by genuine quaternionic matrices.

However, if an observable $O_{\alpha}$ is described by a pure complex Hermitian matrix, its expectation value does not depend on the quaternionic part $j \rho_{\beta}$ of the state $\rho=\rho_{\alpha}+j \rho_{\beta}$.

Now, let us denote by $M(\mathbb{Q})$ and $M(\mathbb{C})$ the space of $n \times m$ quaternionic and complex matrices respectively and let $M=M_{\alpha}+j M_{\beta} \in M(\mathbb{Q})$. We define the complex projection

$$
P: M(\mathbb{Q}) \rightarrow M(\mathbb{C})
$$

by the relation

$$
P[M]=\frac{1}{2}[M-i M i]=M_{\alpha} .
$$

By recalling that the complex projection of a quaternionic density matrix is a complex density matrix [6], we can conclude that the expectation value predicted in the standard $C Q M$ for the state $\rho_{\alpha}$ coincides with the one predicted in $Q Q M$ for the state $\rho$, since

$$
\operatorname{Tr}\left(O_{\alpha} \rho_{\alpha}\right)=\operatorname{Re} \operatorname{Tr}\left(O_{\alpha} \rho_{\alpha}\right)=\operatorname{Re} \operatorname{Tr}\left(O_{\alpha} \rho\right) .
$$

This simple observation is actually very important in our approach, in that it enables us to merge CQM in the (more general) framework of QQM, without modifying any theoretical prediction (as long as complex observables are taken into account).

Moreover, when we consider quaternionic unitary dynamics,

$$
\rho(t)=U(t) \rho(0) U^{\dagger}(t),
$$

where

$$
U(t)=e^{-H t}=U_{\alpha}+j U_{\beta}
$$

with $H=H_{\alpha}+j H_{\beta}=-H^{\dagger}$, the differential equation associated with the time evolution for $\rho$ reads

$$
\frac{d}{d t} \rho(t)=-[H, \rho(t)]
$$

In addition, equations (2) and (3) reduce to

$$
\rho_{\alpha}(t)=U_{\alpha} \rho_{\alpha}(0) U_{\alpha}^{\dagger}+U_{\beta}^{*} \rho_{\alpha}^{*}(0) U_{\beta}^{T}+U_{\alpha} \rho_{\beta}^{*}(0) U_{\beta}^{T}-U_{\beta}^{*} \rho_{\beta}(0) U_{\alpha}^{\dagger}
$$

and

$$
\frac{d}{d t} \rho_{\alpha}=-\left[H_{\alpha}, \rho_{\alpha}\right]+H_{\beta}^{*} \rho_{\beta}-\rho_{\beta}^{*} H_{\beta},
$$

respectively, for the complex projection of the density matrix [6].

It was proven that the dynamics ruled by equation (4) is a one-parameter semigroup dynamics in the space of complex density matrices [6]. 


\section{Pseudo-Hermitian quaternionic density matrices and their dynamics}

In this section, we will introduce the notion of quaternionic pseudo-Hermitian density matrix and a corresponding Liouville-von Neumann type equation will be derived.

Denoting by $Q^{\ddagger}$ the adjoint of an operator $Q$ with respect to the pseudo-inner product

$$
(\cdot, \cdot)_{\eta}=(\cdot, \eta \cdot)
$$

(where $(\cdot, \cdot)$ represent the standard quaternionic inner product in the space $\mathbb{Q}^{n}$ ), we have

$$
Q^{\ddagger}=\eta^{-1} Q^{\dagger} \eta
$$

so that for any $\eta$-pseudo-Hermitian operator, i.e., satisfying the relation,

$$
\eta Q \eta^{-1}=Q^{\dagger},
$$

one has, $Q=Q^{\ddagger}$. These operators constitute the physical observables of the system [13].

If $Q$ is $\eta$-pseudo-Hermitian, equation (7) immediately implies that $\eta Q$ is Hermitian, so that the expectation value of $Q$ in the state $|\psi\rangle$ with respect to the pseudo-inner product (5) can be obtained,

$$
\langle\psi|\eta Q| \psi\rangle=\operatorname{Re} \operatorname{Tr}(|\psi\rangle\langle\psi| \eta Q)=\operatorname{Re} \operatorname{Tr}(\tilde{\rho} Q),
$$

where $\tilde{\rho}=|\psi\rangle\langle\psi| \eta$.

More generally, if $\rho$ denotes a generic quaternionic (Hermitian, positive definite) density matrix, we can associate it with a generalized density matrix $\tilde{\rho}$ by means of a one-to-one mapping in the following way:

$$
\tilde{\rho}=\rho \eta
$$

and obtain $\langle Q\rangle_{\eta}=\operatorname{Re} \operatorname{Tr}(\tilde{\rho} Q)$.

Note that $\tilde{\rho}$ is $\eta$-pseudo-Hermitian:

$$
\tilde{\rho}^{\dagger}=\eta \rho=\eta \tilde{\rho} \eta^{-1} .
$$

As in the Hermitian case discussed in the previous section, equation (8) immediately implies that the expectation value of an $\eta$-pseudo-Hermitian operator $Q$ on the generalized state $\tilde{\rho}$ depends on the quaternionic parts of $Q$ and $\tilde{\rho}$, only if both the operator and the generalized state are represented by genuine quaternionic matrices. Hence, if a $\eta$-pseudo-Hermitian operator $Q$ is described by a complex matrix, its expectation value does not depend on the quaternionic part $j \tilde{\rho}_{\beta}$ of the generalized state $\tilde{\rho}=\tilde{\rho}_{\alpha}+j \tilde{\rho}_{\beta}$.

It was shown that whenever the quaternionic Hamiltonian $H$ of a quantum system is $\eta$ pseudoanti-Hermitian, i.e.,

$$
\eta H \eta^{-1}=-H^{\dagger}
$$

where $\eta=\eta^{\dagger}$, the pseudo-inner product (5), is invariant under the time translation generated by $H$ [10] (we recall that on complex spaces this peculiarity holds also if the Hamiltonian is time dependent but quasi-stationary [14]).

Let a Hermitian nonsingular quaternionic operator $\eta$ be given. Then, the more general $\eta$ pseudoanti-Hermitian quaternionic Hamiltonian $H$ can be written in the following way:

$$
H=A \eta,
$$

where $A^{\dagger}=-A$. 
In fact, let $H$ be given. Then, from the invertibility of $\eta$ the solution of equation (10) can be immediately computed:

$$
A=H \eta^{-1} \text {. }
$$

Now, from the $\eta$-pseudoanti-Hermiticity of $H$ we get

$$
A^{\dagger}=\eta^{-1} H^{\dagger}=-\eta^{-1} \eta H \eta^{-1}=-H \eta^{-1}=-A .
$$

Let us consider now the time evolution of a pure state. Whenever the Hamiltonian $H$ is $\eta$-pseudoanti-Hermitian, the evolution operator $V(t)=e^{-H t}$ :

$$
|\psi(t)\rangle=V(t)|\psi(0)\rangle
$$

is no longer unitary, but $\eta$-unitary, i.e., it satisfies

$$
V^{\dagger} \eta V=\eta
$$

From equations (11) and (12), by easy calculations, we obtain

$$
|\psi(t)\rangle\langle\psi(t)|\eta=V(t)| \psi(0)\rangle\left\langle\psi(0)\left|V^{\dagger}(t) \eta=V(t)\right| \psi(0)\right\rangle\langle\psi(0)| \eta V^{-1}(t),
$$

or, equivalently,

$$
\rho(t) \eta=\tilde{\rho}(t)=V(t) \tilde{\rho}(0) V(t)^{-1},
$$

from which the conservation of the $\eta$-pseudo-norm immediately follows:

$$
\operatorname{Re} \operatorname{Tr} \tilde{\rho}(t)=\operatorname{Re} \operatorname{Tr} \tilde{\rho}(0) .
$$

More generally, the time evolution of $\rho(t)$ is described by the equation

$$
\frac{d}{d t} \rho(t)=-\left(H \rho-\rho H^{\dagger}\right)
$$

$(\hbar=1)$, whereas the time evolution of a generalized density matrix (if $\eta$ is time independent) is described by the usual Liouville-von Neumann equation:

$$
\frac{d}{d t} \tilde{\rho}(t)=-[H, \tilde{\rho}]
$$

\section{Complex projection of quasianti-Hermitian quaternionic Hamiltonian dynamics}

In this section, we restrict ourselves to consider the space of quasi-Hermitian density matrices, that is the subclass of $\eta$-pseudo-Hermitian density matrices where $\eta=B^{\dagger} B$ for some nonsingular bounded operator $B$.

An important property of such generalized density matrices is that they are positive definite; indeed, putting $\eta=B^{\dagger} B$ into equation (9), from the positivity of $\rho$ we immediately obtain $B \tilde{\rho} B^{-1}=B \rho B^{\dagger} \geq 0[15]$.

Then, in this case a new positive definite inner product can be introduced in the Hilbert space where all the usual requirements for a proper quantum mechanical interpretation can be maintained [11, 16, 17, 18].

In order to discuss the complex projection of $\eta$-quasi-Hermitian quaternionic density matrices, we preliminarily recall the following two lemmas [11]: 
Lemma 1. For any Hermitian positive definite $\eta$, the (right) quaternionic Hilbert space $\mathbb{Q}^{n}$ endowed with the scalar product $(\cdot, \cdot)_{\eta}=(\cdot, \eta \cdot)$ is a Hilbert space $\mathbb{Q}_{\eta}^{n}$.

As a consequence of Lemma 1 we can state that any $\eta$-quasi-Hermitian operator in $\mathbb{Q}^{n}$, is Hermitian in $\mathbb{Q}_{\eta}^{n}$. Moreover, as a particular case of the lemma in [11], the following statement holds:

Lemma 2. Let $\{\tilde{\rho}\}$ be an irreducible set of $\eta$-quasi-Hermitian quaternionic operators on the (right) quaternionic Hilbert space $\mathbb{Q}_{\eta}^{n}$ endowed with the scalar product $(\cdot, \cdot)_{\eta}=(\cdot, \eta \cdot)$. Suppose that $\{\tilde{\rho}\}$ cannot be represented in any basis by complex or real operators. Then, the commutant of $\{\tilde{\rho}\}$ is composed of the operators $T=h \mathbf{1}$ (where, $h \in \mathbb{R}$ and $\mathbf{1}$ is the identity operator).

Now, we are able to prove the following proposition, which characterizes the subclass of quaternionic $\eta$-quasi-Hermitian density matrices, $\tilde{\rho}=\rho \eta=\tilde{\rho}_{\alpha}+j \tilde{\rho}_{\beta}$, admitting $\eta$-quasi-Hermitian complex projection density matrices $\tilde{\rho}_{\alpha}$.

Proposition 1. The complex projection $\tilde{\rho}_{\alpha}$ of a $\eta$-quasi-Hermitian quaternionic matrix $\tilde{\rho}=$ $\tilde{\rho}_{\alpha}+j \tilde{\rho}_{\beta}$ is $\eta$-quasi-Hermitian if and only if the entries of $\eta$ are complex.

Proof. By imposing the $\eta$-quasi-Hermiticity of the complex projection of $\tilde{\rho}$, from equation (1) we get:

$$
\eta P[\tilde{\rho}] \eta^{-1}=\frac{1}{2}\left(\tilde{\rho}^{\dagger}-\eta i \eta^{-1} \tilde{\rho}^{\dagger} \eta i \eta^{-1}\right)=P^{\dagger}[\tilde{\rho}]=P\left[\tilde{\rho}^{\dagger}\right]=\frac{1}{2}\left(\tilde{\rho}^{\dagger}-i \tilde{\rho}^{\dagger} i\right),
$$

hence,

$$
\left(\eta^{-1} i \eta i\right) \tilde{\rho}=\tilde{\rho}\left(\eta^{-1} i \eta i\right) .
$$

Now, equation (14) must hold for any quaternionic $\eta$-quasi-Hermitian density matrix $\tilde{\rho}$, and the set $\{\tilde{\rho}\}$ is obviously a quaternionic irreducible set of $\eta$-quasi-Hermitian matrices which cannot be represented in any basis by real or complex operators. Then, Lemma 2 implies

$$
i \eta i=h \eta \quad(h \in \mathbb{R}) .
$$

By using the cyclic property of the real part of the trace and equation (15) we get

$$
\operatorname{Re} \operatorname{Tr}(i \eta i)=\operatorname{Re} \operatorname{Tr}\left(i^{2} \eta\right)=-\operatorname{Re} \operatorname{Tr}(\eta)=\operatorname{Re} \operatorname{Tr}(h \eta)=h \operatorname{Re} \operatorname{Tr}(\eta) .
$$

Now, the positivity of $\eta$ implies, $\operatorname{Re} \operatorname{Tr}(\eta)>0$, hence $h=-1$ and equation (15) becomes

$$
\eta i=i \eta
$$

An important consequence of Proposition 1 is that, if the complex projection $\tilde{\rho}_{\alpha}$ of $\tilde{\rho}$ is $\eta$-quasiHermitian, then, $\tilde{\rho}_{\alpha}$ is positive definite; indeed, $\tilde{\rho}_{\alpha}=\rho_{\alpha} \eta$ where $\eta=B^{\dagger} B$, from the positivity of $\rho_{\alpha}$ we immediately get $B \tilde{\rho}_{\alpha} B^{-1}=B \rho_{\alpha} B^{\dagger} \geq 0$. Hence, from equation (8) we can conclude that if complex $\eta$-quasi-Hermitian operators $Q$ are taken into account, the expectation value of $Q$ on $\tilde{\rho}=\tilde{\rho}_{\alpha}+j \tilde{\rho}_{\beta}$ or on its complex projection $\tilde{\rho}_{\alpha}$ are the same. This observation enables us to merge quasi-Hermitian complex quantum mechanics in the more general framework of quasi-Hermitian quaternionic quantum mechanics, without modifying any theoretical prediction as long as complex observables are taken into account.

Now, we are able to discuss the complex projection of the $\eta$-quasianti-Hermitian quaternionic Hamiltonian dynamics in the space of $\eta$-quasi-Hermitian quaternionic density matrices. Let be given the complex positive definite operator $\eta$. Then, the most general $\eta$-quasianti-Hermitian quaternionic Hamiltonians $H$ reads (see equation (10))

$$
H=H_{\alpha}+j H_{\beta}=\left(A_{\alpha}+j A_{\beta}\right) \eta
$$


where $A_{\alpha}^{\dagger}=-A_{\alpha}$ and $A_{\beta}^{T}=A_{\beta}$. In other words, any $H$ is obtained by adding to a complex $\eta$-quasianti-Hermitian Hamiltonian $A_{\alpha} \eta$ a purely quaternionic $\eta$-quasianti-Hermitian potential term $j A_{\beta} \eta$.

Moreover, putting $\eta=B^{\dagger} B$ into equation (16) we get

$$
H^{\prime}=B H B^{-1}=-\left(B H B^{-1}\right)^{\dagger}=-H^{\prime \dagger}
$$

i.e., if we perform in the Hilbert space $\mathbb{Q}^{n}$ the linear transformation induced by $B$, the Hamiltonian $H^{\prime}$ is anti-Hermitian.

In particular, as said in Section 2, a standard master equation will hold for the complex projection (see equation (4)) of the dynamics generated by $H^{\prime}$

$$
\frac{d}{d t} \rho_{\alpha}^{\prime}=L\left[\rho_{\alpha}^{\prime}\right]=-\left[H_{\alpha}^{\prime}, \rho_{\alpha}^{\prime}\right]+H_{\beta}^{\prime *} \rho_{\beta}^{\prime}-\rho_{\beta}^{*} H_{\beta}^{\prime},
$$

where $\rho_{\alpha}^{\prime}$ and $\rho_{\beta}^{\prime}$ are the complex projection and the purely quaternionic terms, respectively, of the quaternionic (Hermitian) density matrix $\rho^{\prime}=\rho_{\alpha}^{\prime}+j \rho_{\beta}^{\prime}=B \rho \eta B^{-1}=B \rho B^{\dagger}$.

The dynamics ruled by equation (17) is a one-parameter semigroup dynamics in the space of complex (Hermitian) density matrices [6], and we can identify [19, 20]

$$
L\left[\rho_{\alpha}^{\prime}\right]=-\left[H_{\alpha}^{\prime}, \rho_{\alpha}^{\prime}\right]+\sum_{r, s=1}^{n^{2}-1} C_{r s}\left(F_{r}^{\prime} \rho_{\alpha}^{\prime} F_{s}^{\prime \dagger}-\frac{1}{2}\left\{F_{r}^{\prime \dagger} F_{s}^{\prime}, \rho_{\alpha}^{\prime}\right\}\right),
$$

where $F_{r}^{\prime}$ are $n^{2}-1$ traceless square matrices, which form with the normalized identity $F_{0}^{\prime}=$ $I_{n} / \sqrt{n}$ an orthonormal set, i.e., $\operatorname{Tr}\left(F_{r}^{\prime \dagger} F_{s}^{\prime}\right)=\delta_{r s}$, while $\left[C_{r s}\right]$ is a Hermitian matrix.

Then, coming back by means of $B^{-1}:\left|\psi^{\prime}\right\rangle \rightarrow B^{-1}\left|\psi^{\prime}\right\rangle$, we obtain an equation of the Lindblad-Kossakowski type which describes the most general time evolution of the generalized complex projection density matrix $\tilde{\rho}_{\alpha}=\rho_{\alpha} \eta$. In order to write down it explicitly, let us recall that a density matrix transforms as follows: $\rho_{\alpha}^{\prime} \rightarrow B^{-1} \rho_{\alpha}^{\prime}\left(B^{-1}\right)^{\dagger}=\rho_{\alpha}$, so that $\tilde{\rho}_{\alpha}=\rho_{\alpha} \eta=$ $B^{-1} \rho_{\alpha}^{\prime}\left(B^{-1}\right)^{\dagger}\left(B^{\dagger} B\right)=B^{-1} \rho_{\alpha}^{\prime} B$. Moreover, $B^{-1} F_{r}^{\prime} B=F_{r}$ and $B^{-1} F_{s}^{\prime \dagger} B=B^{-1}\left(B F_{s} B^{-1}\right)^{\dagger} B=$ $F_{s}^{\ddagger}$ according to $(6)$, so that, finally [12]:

$$
\frac{d}{d t} \tilde{\rho}_{\alpha}=L\left[\tilde{\rho}_{\alpha}\right]=-\left[H_{\alpha}, \tilde{\rho}_{\alpha}\right]+\sum_{r, s=1}^{n^{2}-1} C_{r s}\left(F_{r} \tilde{\rho}_{\alpha} F_{s}^{\ddagger}-\frac{1}{2}\left\{F_{r}^{\ddagger} F_{s}, \tilde{\rho}_{\alpha}\right\}\right) .
$$

Note that trivially $\operatorname{Tr}\left(F_{r}^{\ddagger} F_{s}\right)=\delta_{r s}$ and the dissipative term

$$
D\left[\tilde{\rho}_{\alpha}\right]=\sum_{r, s=1}^{n^{2}-1} C_{r s}\left(F_{r} \tilde{\rho}_{\alpha} F_{s}^{\ddagger}-\frac{1}{2}\left\{F_{r}^{\ddagger} F_{s}, \tilde{\rho}_{\alpha}\right\}\right)=H_{\beta}^{*} \tilde{\rho}_{\beta}-\tilde{\rho}_{\beta}^{*} H_{\beta},
$$

is quasi-Hermitian:

$$
\eta D\left[\tilde{\rho}_{\alpha}\right] \eta^{-1}=D\left[\tilde{\rho}_{\alpha}\right]^{\dagger}
$$

Finally, the complex projection of quaternionic anti-Hermitian Hamiltonian dynamics can be immediately obtained as a very particular case of this more general setting, putting $\eta=\mathbf{1}$ into equations (18), (19). 


\section{A spin- $\frac{1}{2}$ system in a constant quasianti-Hermitian quaternionic potential}

We will now consider a two-level quantum system with a quasianti-Hermitian quaternionic $H=$ $H_{\alpha}+j H_{\beta}$.

We denote by $H_{\alpha}$ the free complex anti-Hermitian Hamiltonian describing a spin half particle in a constant magnetic field,

$$
H_{\alpha}=\frac{\omega}{2}\left(\begin{array}{cc}
i & 0 \\
0 & -i
\end{array}\right) \text {, }
$$

and by $j H_{\beta}$ a purely quasianti-Hermitian quaternionic constant potential,

$$
j H_{\beta}=\left(\begin{array}{cc}
0 & j \frac{v}{x} \\
j v x & 0
\end{array}\right) \quad(v, x \in \mathbb{R} \backslash\{0\}) .
$$

Note that $H_{\alpha}$ and $j H_{\beta}$ are $\eta$-quasianti-Hermitian, where

$$
\eta=\left(\begin{array}{cc}
x^{2} & 0 \\
0 & 1
\end{array}\right)
$$

The eigenvalues and the corresponding biorthonormal eigenbasis of the quaternionic Hamiltonian $H$ are given by [10]

$$
i E_{ \pm}=i\left(\frac{\omega}{2} \pm v\right)
$$

and

$$
\left|\psi_{ \pm}\right\rangle=\left(\begin{array}{c} 
\pm \frac{i}{x} \\
j
\end{array}\right) \frac{1}{\sqrt{2}}, \quad\left|\phi_{ \pm}\right\rangle=\left(\begin{array}{c} 
\pm x i \\
j
\end{array}\right) \frac{1}{\sqrt{2}} .
$$

The $\eta$-unitary evolution operator reads

$$
\begin{aligned}
V(t) & =e^{-H t}=\left|\psi_{+}\right\rangle e^{-i E_{+} t}\left\langle\phi_{+}|+| \psi_{-}\right\rangle e^{-i E_{-} t}\left\langle\phi_{-}\right| \\
& =\frac{1}{2}\left(\begin{array}{cc}
e^{-i E_{+} t}+e^{-i E_{-} t} & \frac{1}{x}\left(e^{-i E_{-} t}-e^{-i E_{+} t}\right) k \\
x\left(e^{i E_{+} t}-e^{i E_{-} t}\right) k & e^{i E_{+} t}+e^{i E_{-} t}
\end{array}\right) .
\end{aligned}
$$

Let us consider a $\eta$-quasi-Hermitian complex pure initial state:

$$
\tilde{\rho}(0)=\left(\begin{array}{ll}
0 & 0 \\
0 & 1
\end{array}\right)
$$

then, (see equations (13) and (12))

$$
\tilde{\rho}(t)=V(t) \tilde{\rho}(0) V(t)^{-1}=V(t) \tilde{\rho}(0) \eta^{-1} V^{\dagger}(t) \eta=\frac{1}{2}\left(\begin{array}{cc}
1-\cos (2 v t) & -\frac{j}{x} \sin (2 v t) \\
j x \sin (2 v t) & 1+\cos (2 v t)
\end{array}\right) .
$$

The complex projection $\tilde{\rho}_{\alpha}(t)$ of $\tilde{\rho}(t)$ assumes the diagonal form,

$$
\tilde{\rho}_{\alpha}(t)=\frac{1}{2}\left(\begin{array}{cc}
1-\cos (2 v t) & 0 \\
0 & 1+\cos (2 v t)
\end{array}\right) .
$$


The one-parameter semigroup generator associated with the complex projection of the quaternionic $\eta$-unitary dynamics given in equation (21) can be immediately computed (see equations $(18),(19))$ :

$$
L\left[\tilde{\rho}_{\alpha}(t)\right]=-\left[H_{\alpha}, \tilde{\rho}_{\alpha}\right]+H_{\beta}^{*} \tilde{\rho}_{\beta}-\tilde{\rho}_{\beta}^{*} H_{\beta}=\left(\begin{array}{cc}
v \sin (2 v t) & 0 \\
0 & -v \sin (2 v t)
\end{array}\right) .
$$

Let us consider the spin observable, which is associated in QQM with a triple of complex operators. The expectation value of the $z$-component:

$$
s_{z}=\frac{1}{2}\left(\begin{array}{cc}
1 & 0 \\
0 & -1
\end{array}\right) \text {, }
$$

when the system is in the quasi-Hermitian quaternionic pure state (21), is given by (note that $s_{z}$ is $\eta$-quasi-Hermitian contrarily to $s_{x}$ and $s_{y}$ )

$$
\left\langle s_{z}\right\rangle=\operatorname{Re} \operatorname{Tr}\left(s_{z} \tilde{\rho}(t)\right)=\operatorname{Tr}\left(s_{z} \tilde{\rho}_{\alpha}(t)\right)=\frac{\cos (2 v t)}{2} .
$$

By a simple calculation the (positive definite) energy $\eta$-quasi-Hermitian observable $|H|$ reads

$$
|H|=\left|\psi_{+}\right\rangle E_{+}\left\langle\phi_{+}|+| \psi_{-}\right\rangle E_{-}\left\langle\phi_{-}\right|=\left(\begin{array}{cc}
\frac{\omega}{2} & -k \frac{v}{x} \\
k x v & \frac{\omega}{2}
\end{array}\right),
$$

and its expectation value is given by

$$
\langle|H|\rangle=\operatorname{Re} \operatorname{Tr}(|H| \tilde{\rho}(t))=\operatorname{Tr}\left(\left|H_{\alpha}\right| \tilde{\rho}_{\alpha}(t)\right)=\frac{\omega}{2}=\operatorname{Re} \operatorname{Tr}(|H| \tilde{\rho}(0)) .
$$

This example may have interesting physical applications because the quaternionic potential $j H_{\beta}$ in equation (20) strongly affects the spin values (see equation (22)) while the system energy is unchanged (see equation (23)).

\section{References}

[1] Birkhoff G., von Neumann J., The logic of quantum mechanics, Ann. Math. 37 (1936), 823-843.

[2] Finkelstein D., Jauch J.M., Schiminovich S., Speiser D., Foundations of quaternion quantum mechanics, J. Math. Phys. 3 (1962), 207-220.

Finkelstein D., Jauch J.M., Schiminovich S., Speiser D., Principle of general Q-covariance, J. Math. Phys. 4 (1963), 788-796.

Finkelstein D., Jauch J.M., Speiser D., Quaternionic representations of compact groups, J. Math. Phys. 4 (1963), 136-140.

[3] Adler S.L., Quaternionic quantum mechanics and quantum fields, Oxford University Press, New York, 1995.

[4] Kossakowski A., Remarks on positive maps of finite-dimensional simple Jordan algebras, Rep. Math. Phys. 46 (2000), 393-397.

[5] Scolarici G., Solombrino L., Complex entanglement and quaternionic separability, in The Foundations of Quantum Mechanics: Historical Analysis and Open Questions (Cesena, 2004), Editors C. Garola, A. Rossi and S. Sozzo, World Scientific, Singapore, 2006, 301-310.

[6] Asorey M., Scolarici G., Complex positive maps and quaternionic unitary evolution, J. Phys. A: Math. Gen. 39 (2006), 9727-9741.

[7] Asorey M., Scolarici G., Solombrino L., Complex projections of completely positive quaternionic maps, Theoret. and Math. Phys. 151 (2007), 735-743.

[8] Asorey M., Scolarici G., Solombrino L., The complex projection of unitary dynamics of quaternionic pure states, Phys. Rev. A 76 (2007), 012111-012117. 
[9] Proceedings of the Ist, IInd, IIIrd, IVth and Vth International Workshops on "Pseudo-Hermitian Hamiltonians in Quantum Physics" in Czech. J. Phys. 54 (2004), no. 1, no. 10, Czech. J. Phys. 55 (2005), no. 9, J. Phys. A: Math. Gen. 39 (2006), no. 32, and Czech. J. Phys. 56 (2006), no. 9, respectively.

[10] Scolarici G., Pseudoanti-Hermitian operators in quaternionic quantum mechanics, J. Phys. A: Math. Gen. 35 (2002), 7493-7505.

[11] Blasi A., Scolarici G., Solombrino L., Alternative descriptions in quaternionic quantum mechanics, J. Math. Phys. 46 (2005), 42104-42111, quant-ph/0407158.

[12] Scolarici G., Solombrino L., Time evolution of non-Hermitian quantum systems and generalized master equations, Czech. J. Phys. 56 (2006), 935-941.

[13] Mostafazadeh A., Batal A., Physical aspects of pseudo-Hermitian and PT-symmetric quantum mechanics, J. Phys. A: Math. Gen. 37 (2004), 11645-11680, quant-ph/0408132.

[14] Mostafazadeh A., Time-dependent pseudo-Hermitian Hamiltonians defining a unitary quantum system and uniqueness of the metric operator, Phys. Lett. B 650 (2007), 208-212, arXiv:0706.1872.

[15] Zhang F., Quaternions and matrices of quaternions, Linenar Algebra Appl. 251 (1997), 21-57.

[16] Sholtz F.G., Geyer H.B., Hahne F.J.W., Quasi-Hermitian operators in quantum mechanics and the variational principle, Ann. Phys. 213 (1992), 74-101.

[17] Blasi A., Scolarici G., Solombrino L., Pseudo-Hermitian Hamiltonians, indefinite inner product spaces and their symmetries, J. Phys. A: Math. Gen. 37 (2004), 4335-4351, quant-ph/0310106.

[18] Mostafazadeh A., Exact PT-symmetry is equivalent to Hermiticity, J. Phys. A: Math. Gen. 36 (2003), 7081-7092, quant-ph/0304080.

[19] Gorini V., Kossakowski A., Sudarshan E.C.G., Completely positive dynamical semigroups and N-level systems, J. Math. Phys. 17 (1976), 821-825.

[20] Lindblad G., On the generators of quantum dynamical semigroups, Comm. Math. Phys. 48 (1976), 119-130. 